\title{
Scattering of Two Spinless Particles in 3D Formulation with Coulomb Admixtures
}

Received: date / Accepted: date

\begin{abstract}
Scattering of two spinless charge particles for simple forces including coulomb admixtures is calculated without partial wave decomposition. The coulomb interaction being taken is of the type of screened coulomb potential. For the forces range are not infinite, the standard scattering theory is applied. The differential and total cross section is calculated and coulomb effects are shown.
\end{abstract}

Keywords 3D Technique · Coulomb Scattering

\section{Introduction}

Coulomb scattering and scattering with coulomb admixtures have long been challenging problems. This is due to the infinite range of the Coulomb interaction. And although Coulomb interactions in practice are always screened, so that conventional nonrelativistic scattering theory can be applied [1, 2], the effective interaction range is still large. Using the standard partial-wave (PW) technique this means one has to sum up very many partial-wave, which can lead to some difficulties in numerical realization, especially if at the same time one also considers processes in higher energy region.

As an alternative to the PW technique is the so called three-dimensional (3D) approach. Since in 3D calculations the basis states are not expanded into partial waves, there is no problem with partial wave summation, since a priori all partial waves are included. Thus, here we propose to use a 3D approach to calculate scattering with Coulomb admixtures. As an example in this first step, we choose a simple system, that is scattering of two bosons. As interaction we take that of the type of the Malfliet-Tjon [3] . We choose to considered a screened Coulomb potential, with the screening factor being an exponential function. We apply a 3D technique for two boson scattering formulated in Ref. [4].

In Section 2 we shortly show the 3D formulation, which is given in details in Ref. [4]. In Section 3 we show some calculations for differential and total cross sections, where we can see some Coulomb effetcs. We summarize in Section 4 .

\section{Formulation}

The Lippmann-Schwinger equation for the $T$-matrix elements based on the 3D basis states $|\mathbf{q}\rangle$ is given as

$$
T\left(\mathbf{q}^{\prime}, \mathbf{q}\right)=V\left(\mathbf{q}^{\prime}, \mathbf{q}\right)+\lim _{\epsilon \rightarrow 0} \int d^{3} q^{\prime \prime} V\left(\mathbf{q}^{\prime}, \mathbf{q}^{\prime \prime}\right) \frac{1}{\frac{q^{2}}{2 m}+i \epsilon-\frac{q^{\prime \prime 2}}{2 m}} T\left(\mathbf{q}^{\prime \prime}, \mathbf{q}\right),
$$

F. Maulida

Departemen Fisika, Universitas Indonesia, Depok 16424, Indonesia

E-mail: cipangimpenan@gmail.com

I. Fachruddin

Departemen Fisika, Universitas Indonesia, Depok 16424, Indonesia 
with $\mathbf{q}$ being the relative momentum, $m$ the reduced mass of the system, $V\left(\mathbf{q}^{\prime}, \mathbf{q}\right)$ and $T\left(\mathbf{q}^{\prime}, \mathbf{q}\right)$ the potential and the $T$-matrix elements. Since the potential and the $T$-matrix elements are scalar functions, i.e.

$$
\begin{aligned}
& V\left(\mathbf{q}^{\prime}, \mathbf{q}\right)=V\left(q^{\prime}, q, \hat{\mathbf{q}}^{\prime} \cdot \hat{\mathbf{q}}\right) \\
& T\left(\mathbf{q}^{\prime}, \mathbf{q}\right)=T\left(q^{\prime}, q, \hat{\mathbf{q}^{\prime}} \cdot \hat{\mathbf{q}}\right),
\end{aligned}
$$

Eq. (11) finally becomes

$$
T\left(q^{\prime}, q, x^{\prime}\right)=V\left(q^{\prime}, q, x^{\prime}\right)+\lim _{\epsilon \rightarrow 0} \int_{0}^{\infty} d q^{\prime \prime} q^{\prime \prime 2} \int_{-1}^{1} d x^{\prime \prime} v\left(q^{\prime}, q^{\prime \prime}, x^{\prime}, x^{\prime \prime}\right) \frac{1}{\frac{q^{2}}{2 m}+i \epsilon-\frac{q^{\prime \prime 2}}{m}} T\left(q^{\prime \prime}, q, x^{\prime \prime}\right),
$$

where $x^{\prime}=\hat{\mathbf{q}}^{\prime} \cdot \hat{\mathbf{q}}, x^{\prime \prime}=\hat{\mathbf{q}}^{\prime \prime} \cdot \hat{\mathbf{q}}$, and

$$
v\left(q^{\prime}, q^{\prime \prime}, x^{\prime}, x^{\prime \prime}\right)=\int d \varphi^{\prime \prime} V\left(q^{\prime}, q^{\prime \prime}, \hat{\mathbf{q}}^{\prime} \cdot \hat{\mathbf{q}}^{\prime \prime}\right) .
$$

We choose for the short-range interaction those of the type of the Malfliet-Tjon potential,

$$
V(r)=V_{R} \frac{\exp \left[-\mu_{R} r\right]}{r}-V_{A} \frac{\exp \left[-\mu_{A} r\right]}{r},
$$

with $V_{R}, V_{A}, \mu_{R}$, and $\mu_{A}$ being some parameters. For the Coulomb interaction we take the screened one, with the screening factor being an exponential function,

$$
V_{C}(r)=V_{C} \frac{\exp \left[-\mu_{C} r\right]}{r},
$$

where $V_{C}$ and $\mu_{C}$ are the Coulomb parameters. The full interaction in momentum representation is, therefore,

$$
V\left(\mathbf{q}^{\prime}, \mathbf{q}\right)=\frac{1}{2 \pi^{2}}\left\{\frac{V_{R}}{\left(\mathbf{q}^{\prime}-\mathbf{q}\right)^{2}+\mu_{R}^{2}}-\frac{V_{A}}{\left(\mathbf{q}^{\prime}-\mathbf{q}\right)^{2}+\mu_{A}^{2}}+\frac{V_{C}}{\left(\mathbf{q}^{\prime}-\mathbf{q}\right)^{2}+\mu_{C}^{2}}\right\} .
$$

For the potential given in Eq. (8) the integration in Eq. (5) can be evaluated analitically. We obtain

$$
\begin{aligned}
v\left(q^{\prime}, q^{\prime \prime}, x^{\prime}, x^{\prime \prime}\right)= & \frac{1}{\pi}\left[\frac{V_{R}}{\sqrt{\left(q^{\prime 2}+q^{\prime \prime 2}-2 q^{\prime} q^{\prime \prime} x^{\prime} x^{\prime \prime}+\mu_{R}^{2}\right)^{2}-4 q^{\prime 2} q^{\prime \prime 2}\left(1-x^{\prime 2}\right)\left(1-x^{\prime \prime 2}\right)}}\right. \\
& -\frac{V_{A}}{\sqrt{\left(q^{\prime 2}+q^{\prime \prime 2}-2 q^{\prime} q^{\prime \prime} x^{\prime} x^{\prime \prime}+\mu_{A}^{2}\right)^{2}-4 q^{\prime 2} q^{\prime \prime 2}\left(1-x^{\prime 2}\right)\left(1-x^{\prime \prime 2}\right)}} \\
& \left.+\frac{V_{C}}{\sqrt{\left(q^{\prime 2}+q^{\prime \prime 2}-2 q^{\prime} q^{\prime \prime} x^{\prime} x^{\prime \prime}+\mu_{C}^{2}\right)^{2}-4 q^{\prime 2} q^{\prime \prime 2}\left(1-x^{\prime 2}\right)\left(1-x^{\prime \prime 2}\right)}}\right] .
\end{aligned}
$$

\section{Result and discussion}

Here we show some calculations for differential and total cross section. As parameters we take $V_{R}=$ 7.291, $\mu_{R}=613.69 \mathrm{MeV}, V_{A}=-3.1769, \mu_{A}=305.86 \mathrm{MeV}$, and $V_{C}=0.0073$.

First, we observ some Coulomb effects in differential cross section, while varying the range of the Coulomb interaction. As large interaction range as well as high scattering energy require more integration points, especially that for the integration over the momentum's magnitude, we firstly find the optimal number of integration points for very large Coulomb range corresponding to $\mu_{C}=10 \mathrm{MeV}$ and energy of $1000 \mathrm{MeV}$. The obtained number of integration points can then be safely used for smaller Coulomb range and lower energies. In Fig. [1 we show differential cross section for $E_{l a b}=500 \mathrm{MeV}$ and various Coulomb range represented by the Coulomb parameter $\mu_{C}$. Coulomb effects are observed in forward direction. Figure 1 also shows that sufficiently large Coulomb range has to be considered in order to investigate Coulomb effects. For the system being considered varying the Coulomb range 
Differential Cross Section at $E_{l a b}=500 \mathrm{MeV}$

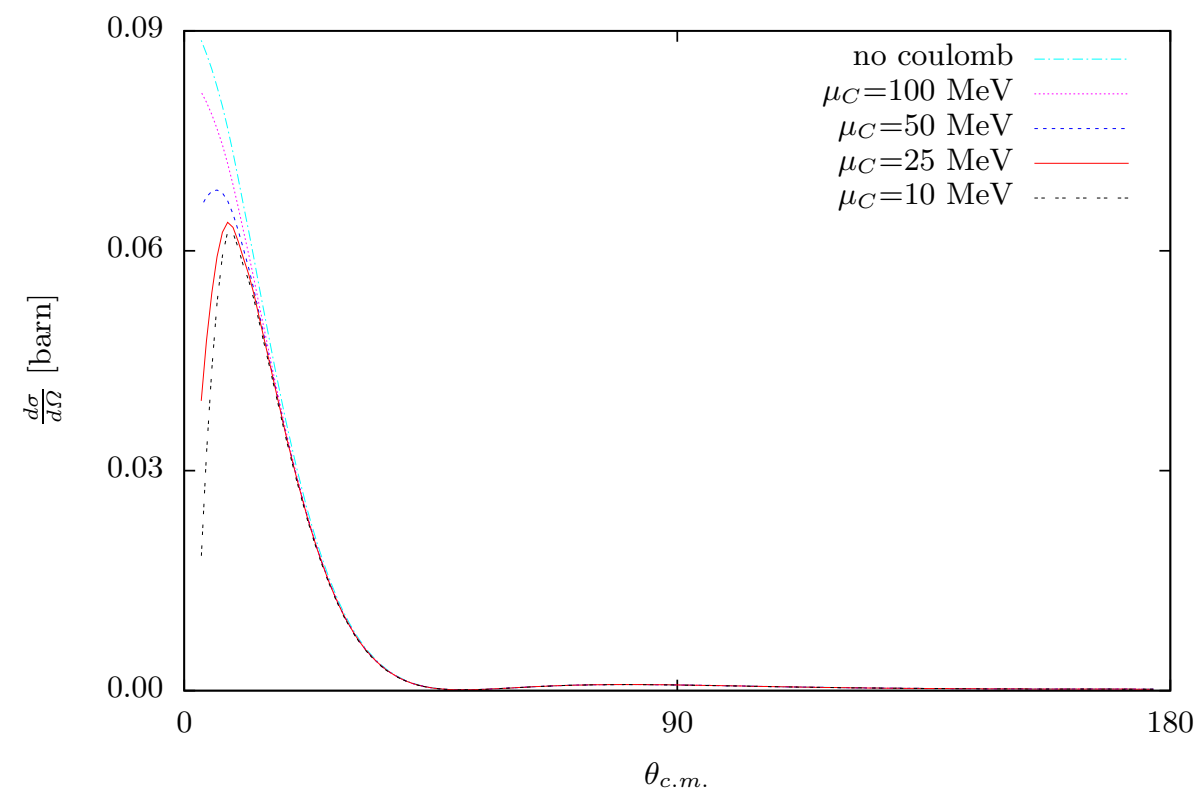

Fig. 1 (color online) Differential cross section for various range of Coulomb interaction represented by $\mu_{C}$.

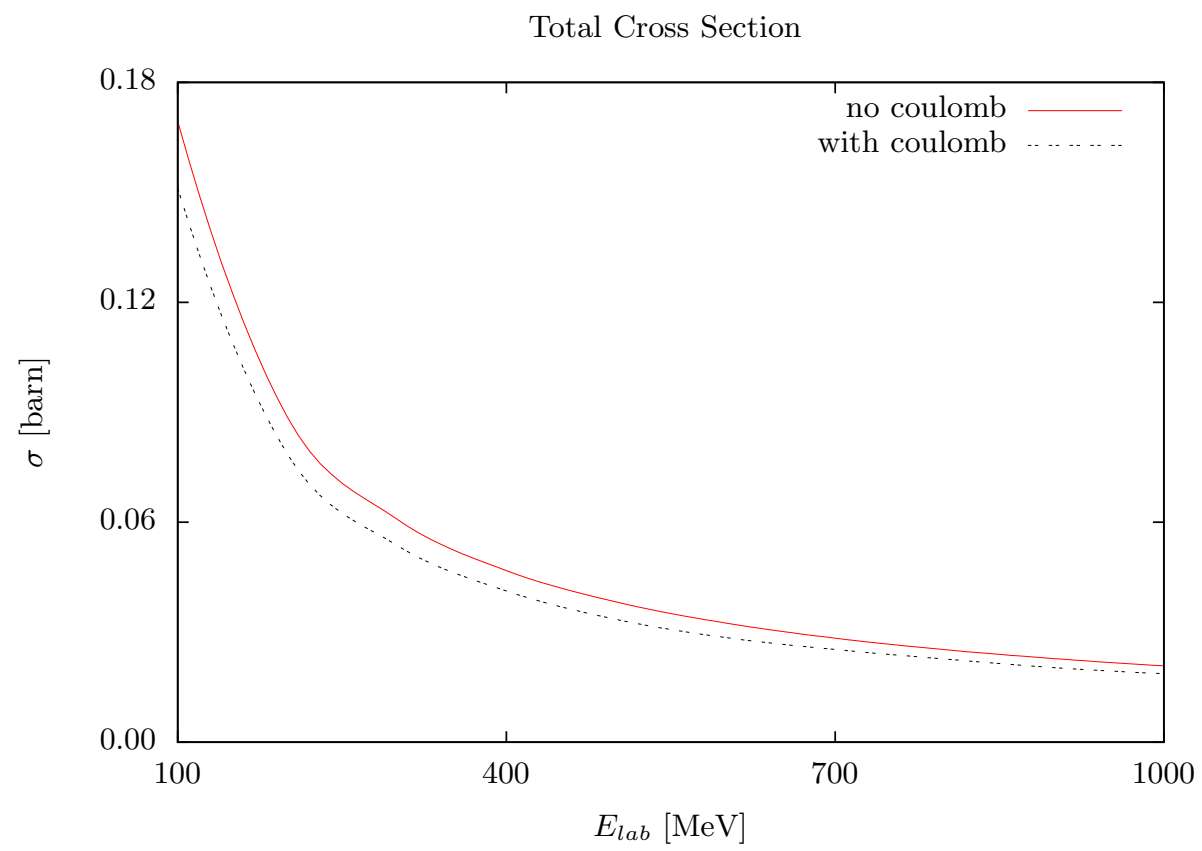

Fig. 2 (color online) Total cross section for various energies, with $\mu_{C}=10 \mathrm{MeV}$ for the Coulomb interaction.

together with applying the $3 \mathrm{D}$ technique leads to no difficulty. We consider that $\mu_{C}=10 \mathrm{MeV}$ is sufficient to represent some kind of effective Coulomb range.

Next we observ Coulomb effects in total cross section for various energies up to $E_{l a b}=1000 \mathrm{MeV}$. We take $\mu_{C}=10 \mathrm{MeV}$. These Coulomb effects are shown in Fig. 2, Including Coulomb interaction lowers the total cross section, especially for lower energies. Varying energy to a high one together with using the 3D technique also leads to no difficulty, at least for the system being considered. 


\section{Summary}

We have demonstrated the use of a 3D technique to calculate scattering of two bosons with Coulomb admixtures. In observing some Coulomb effects we find it not difficult to vary the Coulomb range as well as the energy without much affecting the numerical parameters. For the system being considered $\mu_{C}=10 \mathrm{MeV}$ is sufficient to represent some kind of effective Coulomb range.

\section{References}

1. Taylor, J.R.: Nuovo Cimento 23B, 313 (1974)

2. Semon, M.D., Taylor, J.R.: Nuovo Cimento 26B, 48 (1975)

3. Malfliet, R.A., Tjon, J.A.: Nucl. Phys. A 127, 161-168 (1969)

4. Elster, Ch., Thomas, J.H., Glöckle, W.: Few-Body Systems 24, 55-79 (1998) 